\title{
EVIDENCE FOR RAPID MOTION OF THE OUTER BOUNDARY OF THE MAGNETOSPHERE
}
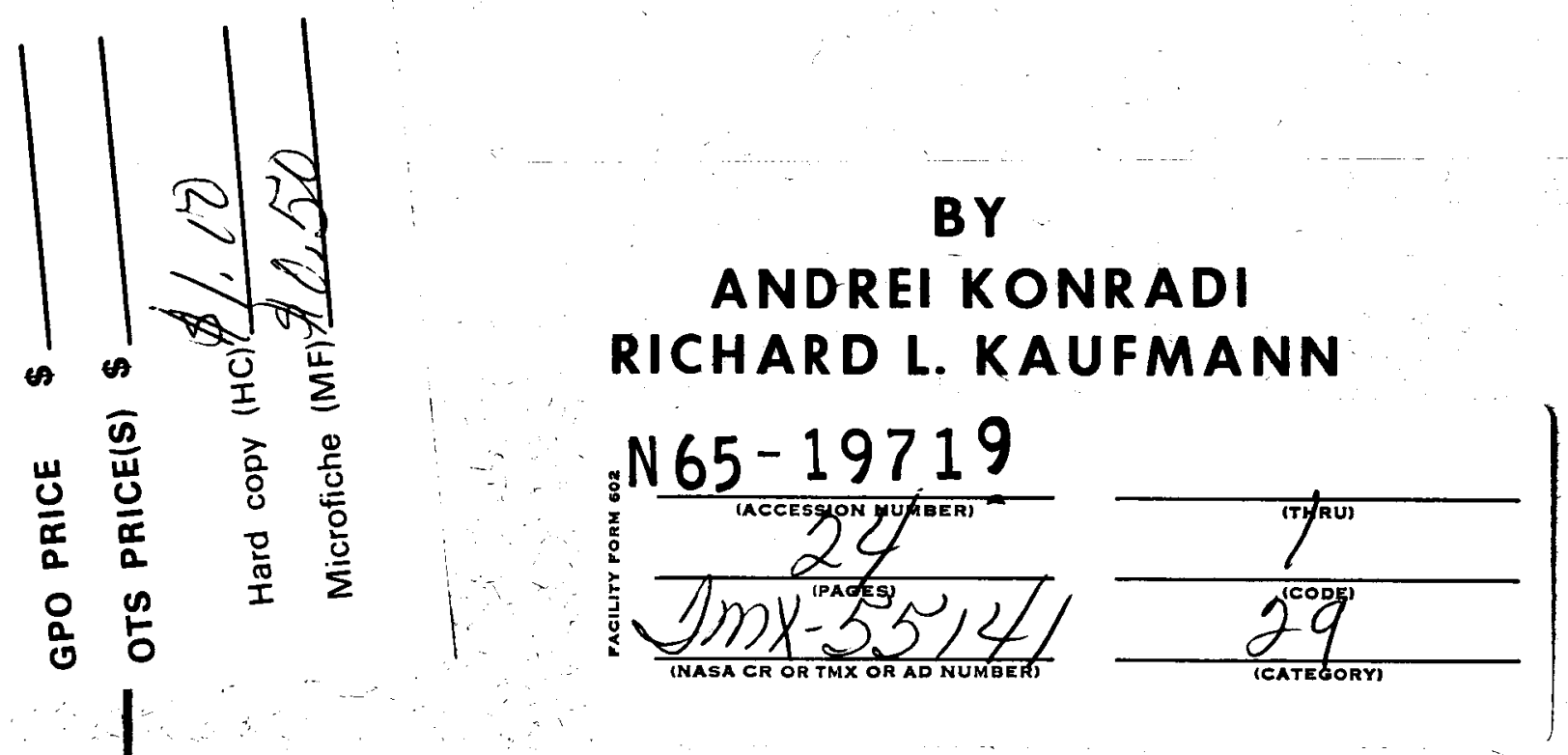

OCTOBER 1964

NASA

GODDARD SPACE FLIGHT CENTER GREENBELT, MARYLAND 
EVIDENCE FOR RAPID MOTION OF THE OUTER

BOUNDARY OF THE MAGNETOSPHERE

\author{
Andrei Konradi \\ NASA-Goddard Space Flight Center \\ Greenbelt, Maryland \\ and \\ Richard L. Kaufmann \\ University of New Hampshire \\ Durham, New Hampshire
}




\title{
EVIDENCE FOR RAPID MOTION OF THE OUTER \\ BOUNDARY OF THE MAGNETOSPHERE
}

\author{
by \\ Andrei Konradi \\ NASA-Goddard Space Flight Center \\ Greenbelt, Maryland \\ and \\ Richard L. Kaufmann \\ University of New Hampshire \\ Durham, New Hampshire
}

\section{ABSTRACT}

\section{9}

A sudden increase of the particle intensity and of the magnetic field strength on September 25, 1961 at 17:31 U.T. was detected by the ion-electron detector and the magnetometer on board Explorer XII outside the earth's magnetosphere. From the physical extent of the region and the energy of the trapped protons the temporal nature of the event was established. Similarities between particle fluxes as well as between magnetic fields during the increase and within the outer belt make it probable that the increase represents an impulse-like extension of at least a portion of the outer belt. A deduced lower limit on the outward radial velocity of the extension is $156 \mathrm{~km} / \mathrm{sec}$. Crude calculations show that either the proton density near the outer edge is less than $100 \mathrm{~cm}^{-3}$ or the temperature is greater than $15 \mathrm{ev}$.

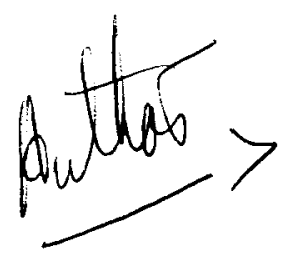


CONTENTS

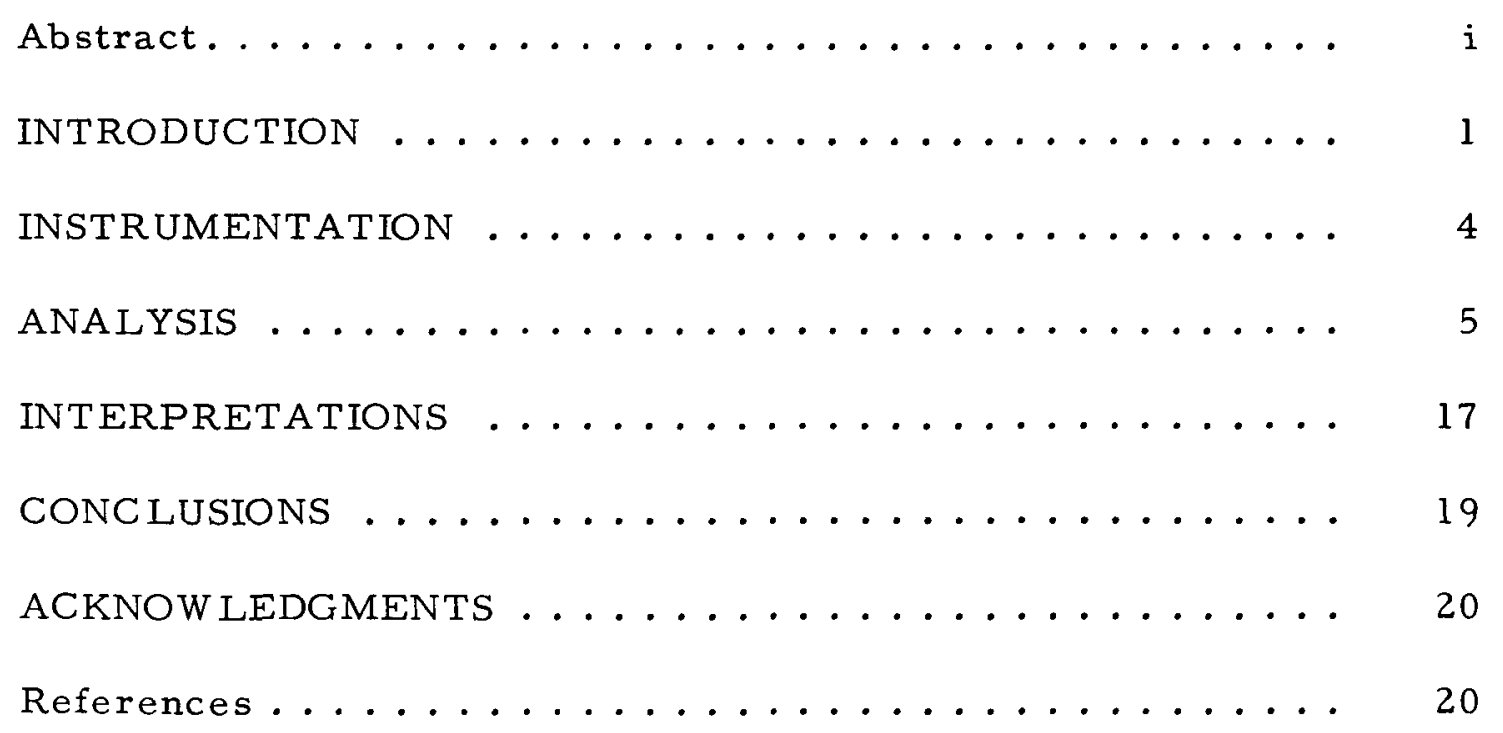




\author{
EVIDENCE FOR RAPID MOTION OF THE OUTER \\ BOUNDARY OF THE MAGNETOSPHERE \\ by \\ Andrei Konradi \\ NASA-Goddard Space Flight Center \\ Greenbelt, Maryland \\ and \\ Richard L. Kaufmann \\ University of New Hampshire \\ Durham, New Hampshire
}

\title{
INTRODUCTION
}

The Explorer XII satellite was well suited for the study of the outer boundary of the trapping region. With an apogee of over $13 R_{e}$ (earth's radii) it usually penetrated the boundary on the sunward side twice during each orbit. The position of the boundary ranged from about $8 \mathrm{R}_{e}$ to beyond the apogee of the satellite with an average value of somewhere about $10 \mathrm{R}_{\mathrm{e}}$ (Cahill and Amazeen, 1963).

Data from the ion-electron detector flown by Davis and Williamson and from the magnetometer flown by Cahill on board Explorer XII has yielded more than 100 transitions of the outer boundary. For our purposes a transition shall be defined to occur when on an inbound pass the output current of the photomultiplier, measured in the mode which is most sensitive to the incident energy flux, rises significantly above the dark current (usually at least by an order of magnitude) in a time interval of the order of minutes corresponding to a radial distance of about one hundred kilometers covered by the satellite. The reverse is of course true for an outbound pass (Davis and Williamson, 1963). Similarly, a transition of the boundary is indicated by the magnetometer when there is usually an abrupt change in either both the magnitude and direction of the magnetic field, or just a change in the direction of the magnetic field. Several typical transitions are shown in Figures la 
OUTER EDGE OF THE EARTH'S MAGNETOSPHERE
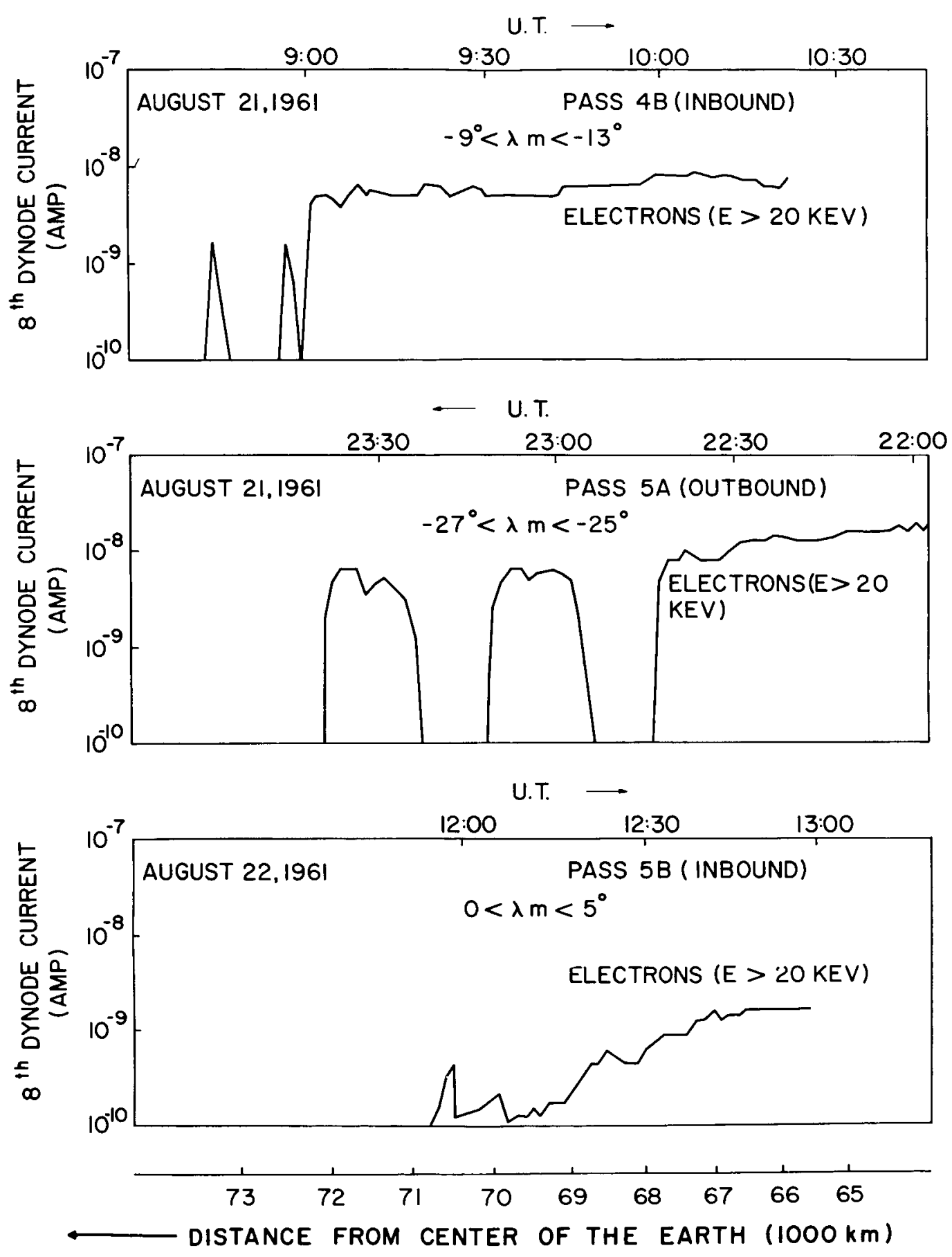

Figure la-Three samples of Explorer XII transitions of the outer boundary of the trapping region as seen by the ion-electron detector. The 8th dynode current is a measure of the electron intensity for electrons with $20 \mathrm{kev}<\mathrm{E}<1$ Mev. 

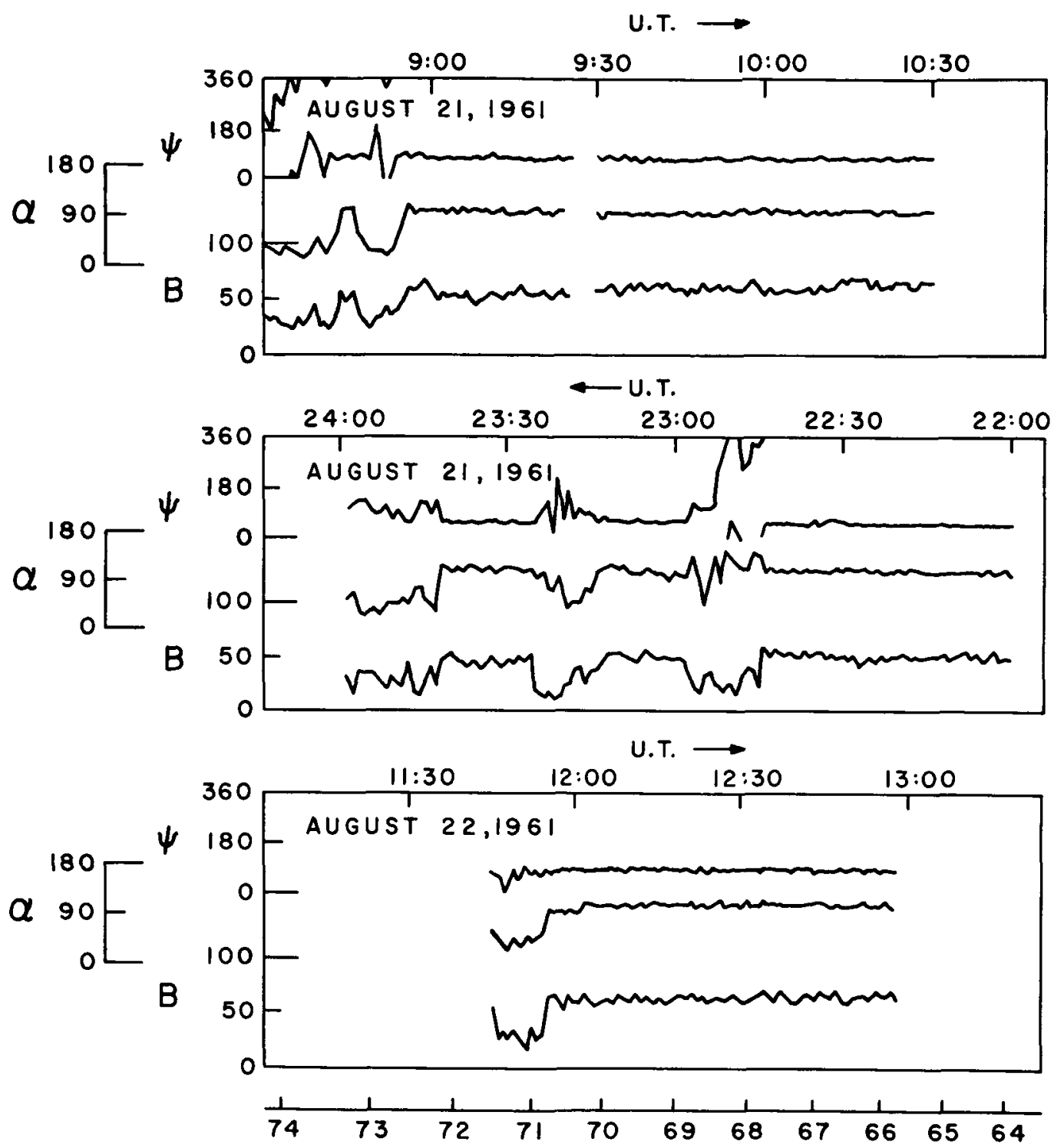

— DISTANCE FROM CENTER OF THE EARTH(1000 km)

Figure lb-Magnetic field directions and intensity for the same transitions as in Figure la. The strength is measured in gammas and the directions are given in the satellite centered spherical coordinates $\alpha$ and $\psi$.

and $\mathrm{lb}$. As can be seen from Figure la, at times the satellite passes through regions of enhanced particle intensity which are separated from the boundary by stretches where the particle intensity falls below the threshold of detectability. Unfortunately, in most cases it is impossible 
to separate temporal and spatial phenomena on the basis of only particle data. One could argue equally well that the intensity represent actually a part of the outer belt that for some reason is separated from the rest of the belt by particle free regions, or that they represent an expansion and contraction of the trapping region in such a way that the satellite crosses the boundary several times during one orbit. Figure $1 \mathrm{~b}$ shows the magnitude and direction of the magnetic field during the same transitions. The directions of the magnetic field are given in satellite centered spherical coordinates (Cahill and Amazeen 1963). It should be noted that in analogy to particle intensities during the transition of the boundary the magnetic field sometimes shows the same direction and magnitude in regions separated from the magnetosphere as were observed in the trapping region. Using magnetic field data alone one again cannot distinguish between temporal and spatial phenomena.

In the analysis of the data thus far, one case was found in which a combination of magnetic field data and particle data can resolve this question.

The present paper shall be concerned with a sudden increase of the particle flux on September 25, 1961 at 17:31 U.T. at a radial distance of $77,465 \mathrm{~km}$ as seen by the ion-electron detector and changes in the magnetic field as seen by the magnetometer on board Explorer XII.

\section{INSTRUMENTATION}

A brief review of some of the features of the ion-electron detector pertinent to the following discussion will be given here. A detailed description of the apparatus will be published elsewhere (Davis and Williamson, to be published).

The detector is a scintillation counter consisting of a photomultiplier tube on the face of which is deposited a $5 \mathrm{mg} / \mathrm{cm}^{2}$ thick layer of crystalline $\mathrm{ZnS}$ covered by a $1000 \mathrm{~A}^{\circ}$ thick layer of $\mathrm{Al}$.

It has a geometric factor of $5.4 \times 10^{-3} \mathrm{~cm}^{2}$ ster. A collimator allows particles to enter from a viewing cone with a half angle of $11^{\circ}$.

A wheel driven by a stepping motor introduces varying thicknesses of $\mathrm{Ni}$ absorber between the collimator and the phosphor. Three positions on the wheel carry Au discs which scatter the incident flux entering through an alternate collimator into the phosphor. 
There are three modes of operation:

1. Pulse output from the anode measures protons between the energies of $100 \mathrm{kev}$ and $5 \mathrm{Mev}$.

2. 8th dynode current measures the total incident energy flux from both protons and electrons.

3. Electrons can be selected preferentially by scattering an incident beam of both protons and electrons from an Au disc. In this mode of operation the 8th dynode current measures the total incident energy flux due to electrons alone.

In all three modes of operation the lower energy cutoff can be raised by introducing varying thicknesses of $\mathrm{Ni}$ absorber.

Throughout the following discussion we shall make the assumption that for different absorbers we are measuring the integral proton flux above some energy for which the detector efficiency is $50 \%$. The situation is more complicated for electrons because of the shape of the sensitivity curves and the necessity to use approximation methods to obtain electron spectrums.

The magnetic field data was obtained from the magnetometer flown by L. J. Cahill of the University of New Hampshire and its operation had been described earlier (Cahill and Amazeen, 1963).

\section{ANALYSIS}

The inbound pass of September 25, 1961 had a geomagnetic latitude of less than $5^{\circ}$ for radial distances of more than $40,000 \mathrm{~km}$. Figure 2 shows intensities of low energy protons and the value of the photomultiplier 8th dynode current obtained with $5 \mu$ inch $\mathrm{Ni}$ absorber when the detector was looking at particles with local pitch angles of around $90^{\circ}$. Also shown are the intensity and direction of the magnetic field in the satellite coordinate system.

The electrometer data represents instantaneous values of the 8th dynode current sampled at intervals of 83 seconds. To gain some physical idea of the electron intensities involved, a crude analysis shows that at 19:45 U.T. the differential electron spectrum could be approximated by a curve of the form $E^{-\gamma}$ where $E$ is the electron 

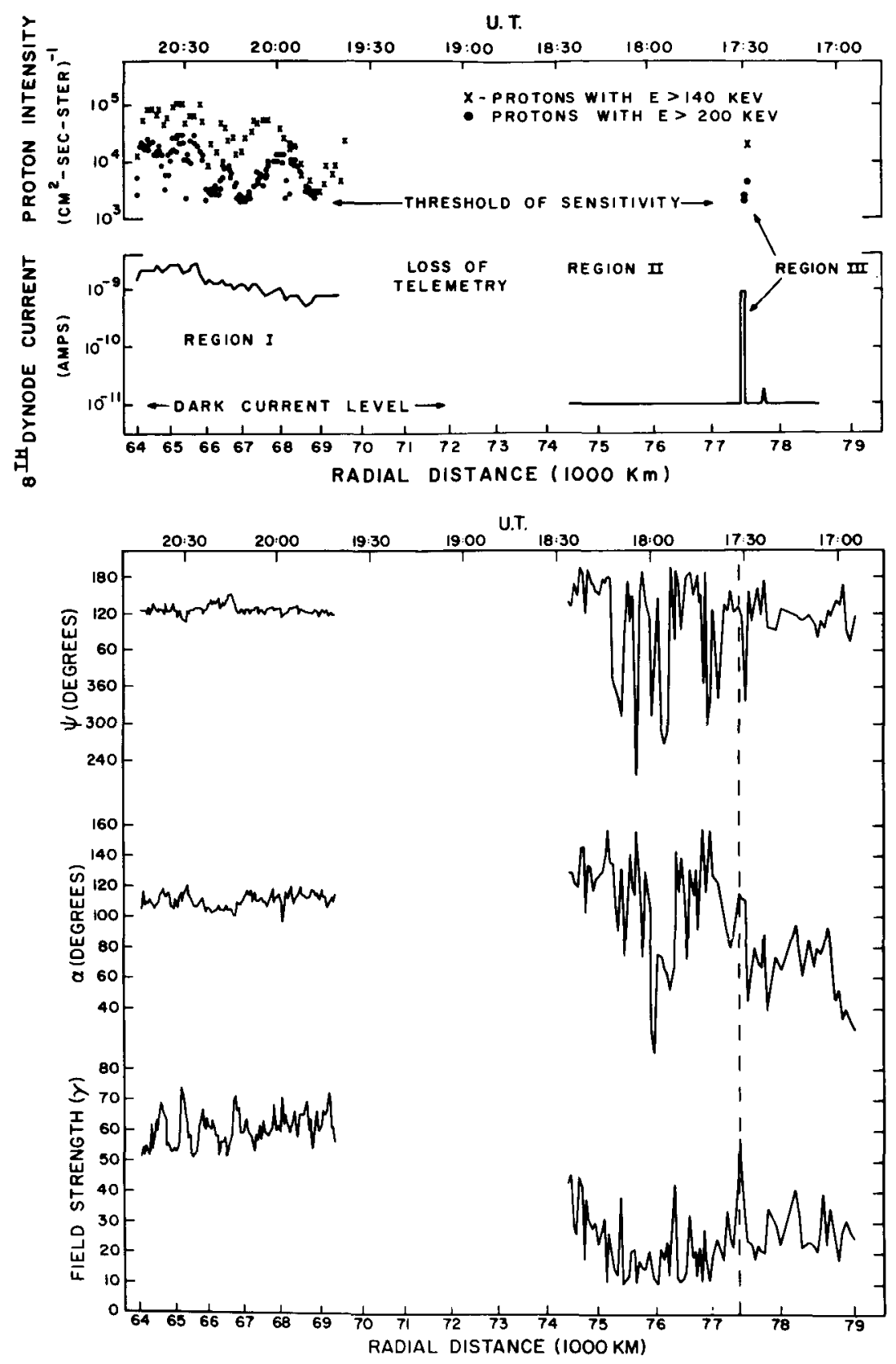

- "

Figure 2-Inbound pass of September 25, 1961. The upper two curves represent the unidirectional proton intensity for particles with $90^{\circ}$ local pitch angle and the 8th dynode current corresponding to electrons with $E>20 \mathrm{kev}$ and $90^{\circ}$ pitch angle respectively. The lower three curves show the strength and directions of the magnetic field. Angles $\alpha$ and $\psi$ were previously defined by Cahill and Amazeen (1963). The left side is a typical response for the trapping region (Region I). The right side (Region II) shows the usual response of the detectors outside the magnetosphere. For protons the threshold of sensitivity is the count rate corresponding to an omnidirectional flux of penetrating particles. Region III is the sudden increase in particle and magnetic field intensity which is the subject of the present study. 
energy and $\gamma=3.7$. The integral intensity of electrons for $E>20 \mathrm{kev}$ is $4 \times 10^{6}$ electrons $\left(\mathrm{cm}^{2} \mathrm{sec} \text { ster }\right)^{-1}$. This number is accurate to better than a factor of 2 .

The plot of the proton data was made for particles with energies $E>140 \mathrm{kev}$ and $\mathrm{E}>200 \mathrm{kev}$. The proton intensities are obtained from data accumulated over a time period of 0.3 seconds. During that time the satellite turns about its axis through an angle of about $58^{\circ}$. Thus the $90^{\circ}$ pitch angle intensity is an average value covering also other than locally mirroring particles.

The magnetometer data corresponds to an average of ten successive telemetry samples taken at intervals of $50 \mathrm{~km}$. The field strength is expressed in gammas, and the direction is indicated in spherical satellite centered coordinates mentioned earlier.

The graph in Figure 2 is divided into three parts: Region I represents the proton and current intensities in the outer belt. Region II is characteristic of the space outside the trapping region. In this case the current output of the detector consists only of the dark current and the pulse output merges into the omnidirectional background. Region III shows a sudden increase in the electron and proton intensity at 17:31 U.T. which lasted for about one minute.

Similarly the magnetic field in Region $I$ is the rather constant field encountered in the outer belt. Region II is typical of the field outside the boundary of the magnetosphere. The field is rather disorganized and shows many changes in direction. The dashed line indicates the magnetic field in Region III. During the transition of Region III the magnetic field agreed in magnitude and in direction with the field observed in Region I. Before and after the sudden increase the magnetic field had an average value of 25 gamma. During the transition of Region III the field rose to 61 gamma. Aside from this single increase there is nothing unusual happening in the behavior of the field outside the magnetospheric boundary. It should be emphasized that in this context the word "region" is used only with reference to the graph and does not necessarily imply any physical structure in space. The increase is real and not due to faulty data processing because it appears on two completely independent data tapes acquired at Fort Myers, Florida and at South Point, Hawaii. This increase in particle and field intensity will from now on be referred to as Region III. 
While the satellite was in Region III it was possible to obtain 15 data points for protons with $E>140 \mathrm{kev}$ and 45 data points for protons with $E>200 \mathrm{kev}$. Figures 3 and 4 present the pitch angle distributions for these two cutoff energies. In both cases the pitch angles were assigned from the knowledge of the direction of the magnetic field as seen by the magnetometer. The vertical bars represent Poisson standard deviations only. The uncertainty due to the digitization of the analog data tapes is about half as large again. In Figure 3 the horizontal bars represent the ranges of pitch angles which were scanned during the accumulation period. The pitch angle assigned to each point is the value seen at the center of the accumulation period. The points represented

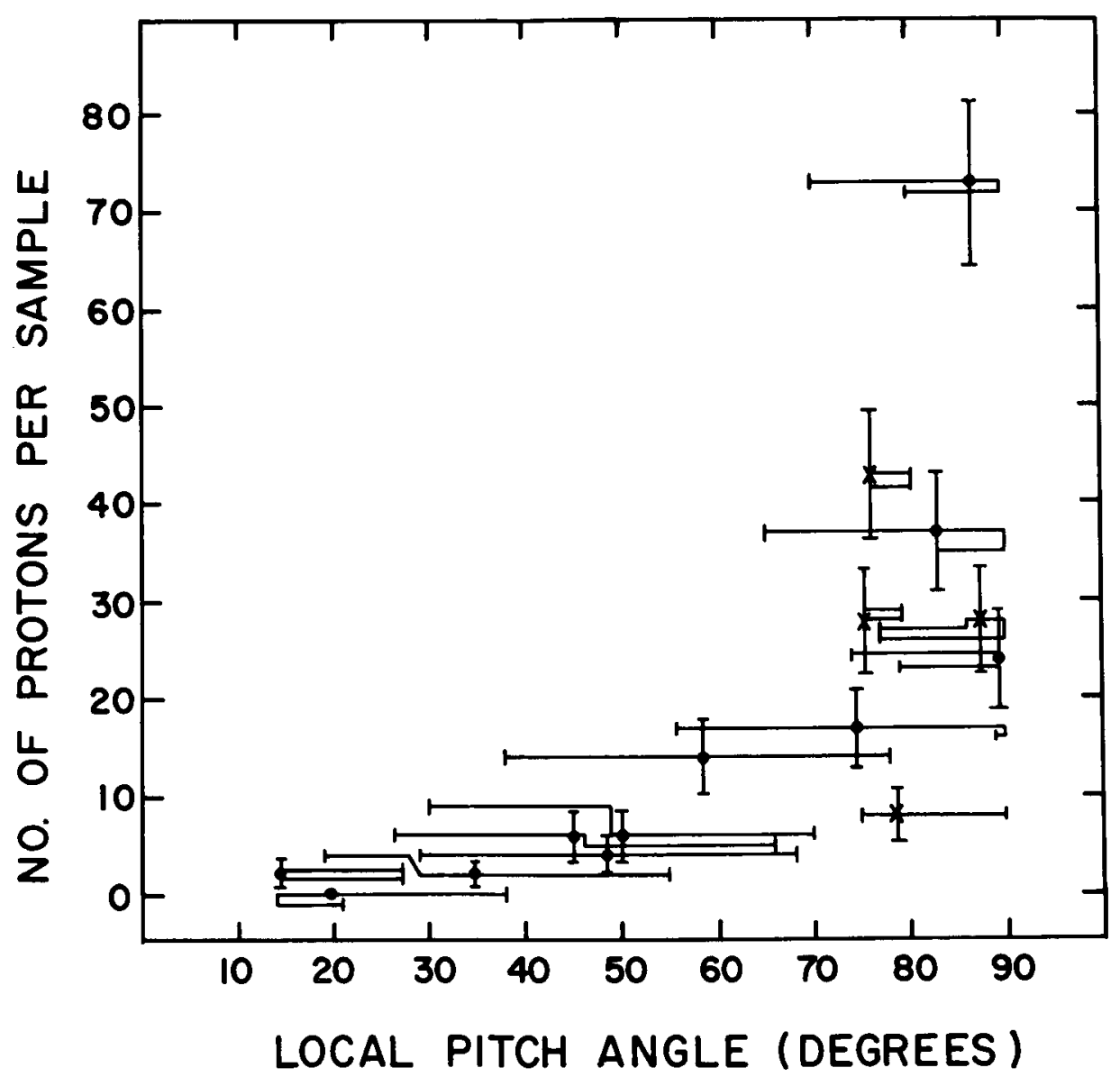

Figure 3-Pitch angle distribution of protons with E > 140 kev found in Region III. The vertical bars represent Poisson standard deviations. The uncertainties due to the digitization of the analog data are about half as large again. The horizontal bars represent the range of angles over which the samples were collected. The assigned p.a. corresponds to the center of the accumulation period. 


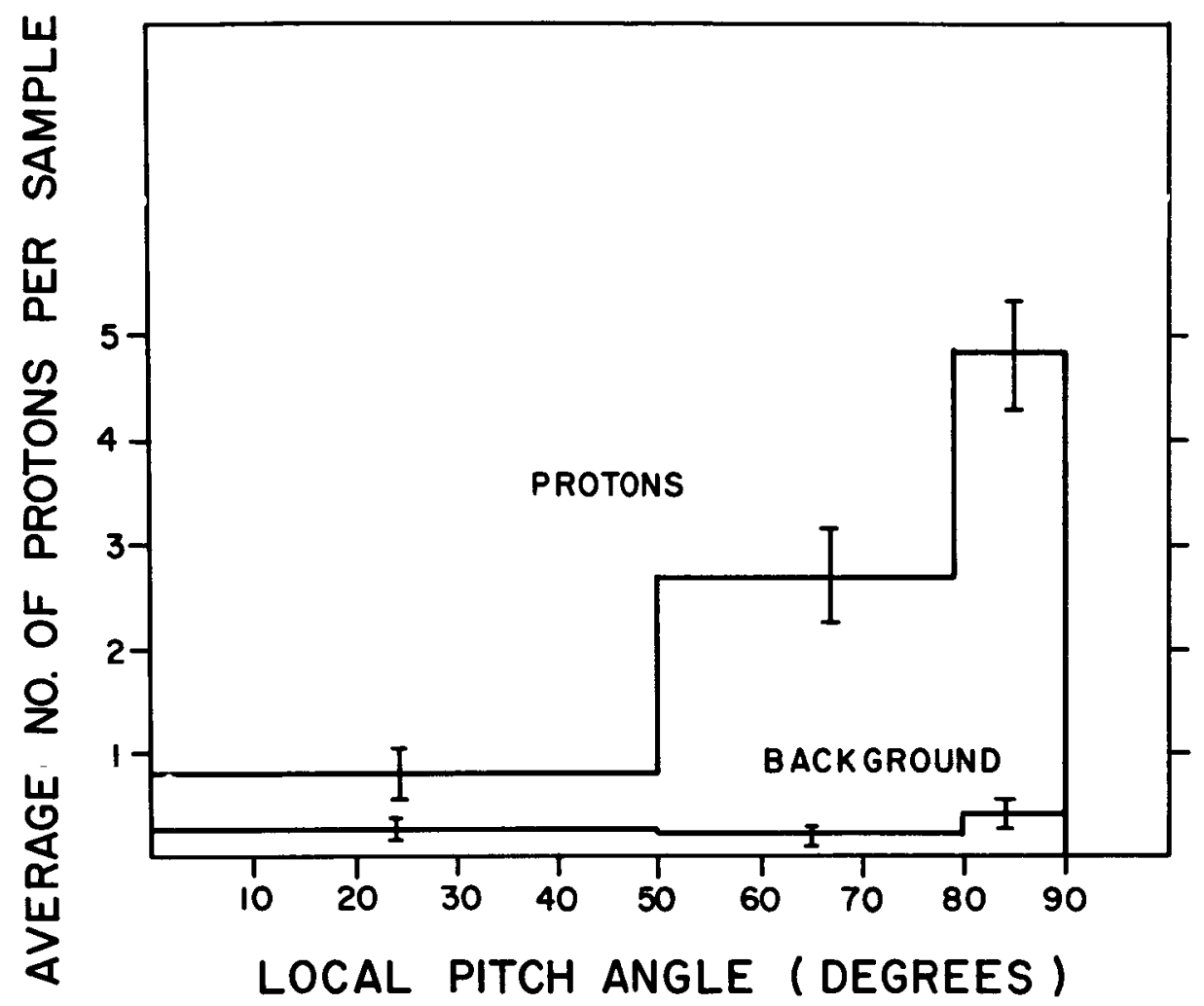

Figure 4-Pitch angle distribution of protons with $E>200 \mathrm{kev}$. The data points were lumped together into three groups each containing 15 points. The vertical bars represent Poisson deviations. The background count obtained with a thick plug in front of the detector aperture is given below.

by crosses actually have pitch angles which are supplements of the indicated pitch angles. Figure 4 is a similar diagram. In this case the number of counts per data point was small so that data points were lumped together into three groups each containing 15 points. The bars again represent Poisson standard deviations. For contrast the omnidirectional background count obtained with a thick plug in front of the detector is plotted below.

The shape of the pitch angle distributions is quite similar to those obtained for trapped particles within the magnetosphere in that the maximum intensity is found at $90^{\circ}$ local pitch angle (Davis and Williamson, 1963). By contrast, solar protons during a sudden commencement have been observed to have maximum intensities in directions other than $90^{\circ}$ to the field lines (Hoffman, et al., 1962). 
Figure 5 shows the magnetic field intensity and directions during the transition of Region III. The vertical bars represent standard deviations of the mean of 16 data points each. The scatter in the original data is mostly due to the digitization of the magnetometer analog output voltage. The standard deviations increase as the field decreases in magnitude. $a$ is the angle between the spin axis of the satellite and the magnetic field. $\psi$ is the angle between the spin-field plane and the spinsun plane. They are identical to those defined earlier by Cahill and Amazeen (1963). Before and after the transition of Region III the magnitude of the field was about 25 gamma. During the transition the magnitude rose to 60 gamma. There was no drastic change in either a or $\psi$.

Assuming for the moment that Region III is stationary an estimate of its maximum width can be made by finding the time when the particle flux was zero on the step corresponding to the lowest energy cutoff just before and after the transition of Region III and multiplying it by the velocity of the satellite. This gives the region a maximum width of $200 \mathrm{~km}$. A more precise value obtained from the magnetometer data is $83 \mathrm{~km}$ corresponding to a width of $56 \mathrm{~km}$ in the radial direction from the center of the earth. It should be pointed out that the angle between the direction of the magnetic field and the velocity vector of the satellite is about $60^{\circ}$. This means that the angle between the velocity vector and the plane of gyration of protons with $90^{\circ}$ pitch angle is $30^{\circ}$. Therefore, the width of Region III in the plane containing protons with $90^{\circ}$ pitch angles must be close to that of Region III as measured along the velocity vector.

Table I shows the cyclotron radii of protons with several energies corresponding to the magnetic field encountered within Region III. It is clear from the Table that no $200 \mathrm{kev}$ or $140 \mathrm{kev}$ protons with pitch angles close to $90^{\circ}$ could be trapped within a stationary Region III where the magnetic field strength is about 60 gamma. The efficiency of the detector for $310 \mathrm{ev}$ protons is zero. Thus the conclusion must be drawn that Region III is a temporal rather than a spatial phenomenon.

The origin of Region III can only be conjectured. In what follows we shall try to produce convincing evidence that Region III represents a temporal extension of the outer belt.

Comparison between Region I and Region III shows that the intensities of both the 8th dynode current and of the protons are comparable in several places for particles with $90^{\circ}$ pitch angle. The order of magnitude fluctuations of the proton flux and the somewhat smaller 

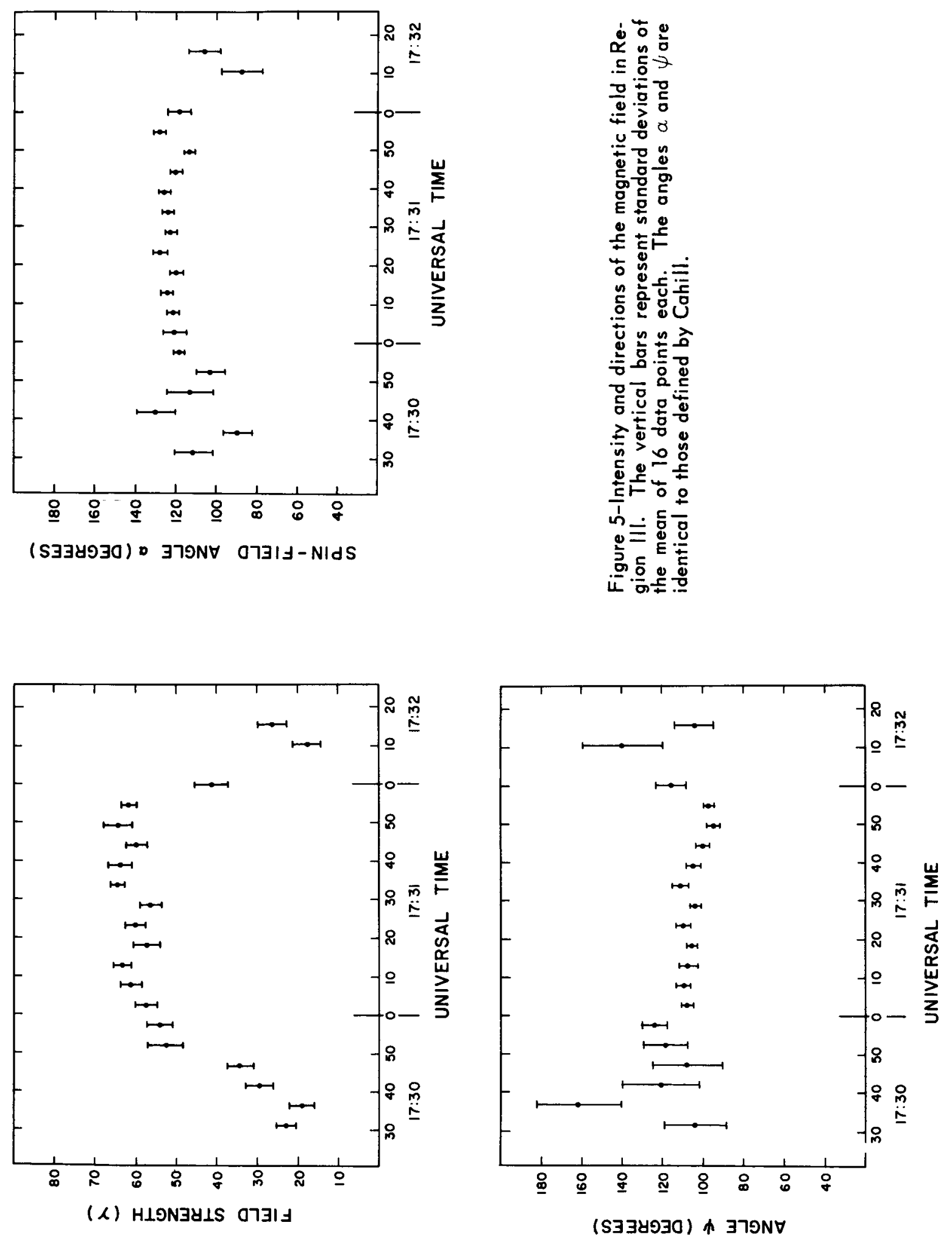
Table I

Cyclotron Radii of Protons

\begin{tabular}{|c|c|}
\hline \multicolumn{2}{|c|}{ Magnetic field strength $\mathrm{H}=60$ gamma } \\
\hline Proton energy & Cyclotron radius \\
\hline $200 \mathrm{kev}$ & $1075 \mathrm{~km}$ \\
$140 \mathrm{kev}$ & $899 \mathrm{~km}$ \\
$310 \mathrm{ev}$ & $42 \mathrm{~km}$ \\
\hline
\end{tabular}

fluctuations of the 8th dynode current make it difficult to perform any more detailed analysis. Magnetometer data, however, provide a more quantitative comparison.

For reasons of clarity, we shall transform the angles $\alpha$ and $\psi$ into the geomagnetic coordinate system.

To make a comparison with the directions of the geomagnetic field within the radiation belt, the direction of the field was obtained from experimental data at three points shown in Table II. Region Ia occurs just after the recovery of the telemetry within the belt. Region Ib was selected to have the same increment of distance as exists between III and Ia.

Table II

\begin{tabular}{|c|c|c|}
\hline Time (U.T.) & Region & Radial distance (earth radii) \\
\hline $17: 31$ & III & 12.135 \\
$19: 42$ & Ia & 10.877 \\
$21: 12$ & Ib & 9.618 \\
\hline
\end{tabular}

Several theoretical models indicate that in the absence of a ring current and close to the equatorial plane the boundary of the magnetosphere is almost circular in the meridian plane on the day side of the earth. (Beard, 1960; Beard, 1962; Beard and Jenkins, 1962; Davis and Beard, 1962; Mead and Beard, 1964; Spreiter and Briggs, 1962; Spreiter and Alksne, 1962; Spreiter and Hyett, 1963). Assuming that this holds 
true the direction of the magnetic field was calculated just within the boundary of the magnetosphere at $+4^{\circ}$ geomagnetic latitude and at 10:24 hours local time.

The results of the field calculations and observations are given in Table III.

Table III

Directions of the Magnetic Field

\begin{tabular}{|c|c|c|c|c|c|c|c|c|}
\hline \multirow{2}{*}{ Region } & \multirow{2}{*}{ Time } & \multirow{2}{*}{ Field $(\gamma)$} & \multirow{2}{*}{\multicolumn{2}{|c|}{$\psi($ deg.) }} & \multirow{2}{*}{$\alpha$ (deg.) } & \multicolumn{3}{|c|}{$\begin{array}{l}\text { Field Geomagnetic } \\
\text { Coord. }\end{array}$} \\
\hline & & & & & & $\begin{array}{l}\text { Lat. } \\
\text { (deg.) }\end{array}$ & $\begin{array}{l}\text { Long. } \\
\text { (deg). }\end{array}$ & $\begin{array}{c}\phi \\
\text { (deg.) }\end{array}$ \\
\hline III calc. & $17: 31$ & & \multirow{4}{*}{\multicolumn{2}{|c|}{$\begin{array}{l}113.45 \\
105.0 \pm 1.0 \\
116.3 \pm 0.7 \\
119.5 \pm 0.9\end{array}$}} & 126.4 & 86.06 & 139.81 & 180.00 \\
\hline III obs. & $17: 31$ & $61.1+0.8$ & & & $122.4 \pm 0.8$ & \begin{tabular}{l|l}
8 & 83.92
\end{tabular} & -115.50 & -75.69 \\
\hline Ia obs. & $19: 41$ & $71.5 \pm 0.7$ & & & $120.4 \pm 0.6$ & \begin{tabular}{l|l|l}
6 & 81.11
\end{tabular} & 179.82 & -112.53 \\
\hline Ib obs. & $21: 12$ & $73.5 \pm 1.1$ & & & $118.2 \pm 0.9$ & 979.92 & 166.54 & -107.12 \\
\hline \multicolumn{9}{|c|}{ Positions of the Sun and Satellite } \\
\hline \multirow{2}{*}{ Region } & \multirow{2}{*}{ Time } & \multicolumn{4}{|c|}{ Satellite Geom. Coord. } & \multicolumn{3}{|c|}{ Sun Geom. Coord. } \\
\hline & & \multicolumn{2}{|c|}{ Lat. (deg.) } & \multicolumn{2}{|c|}{ Long. (deg.) } & Lat. (deg.) & \multicolumn{2}{|c|}{ Long. (deg.) } \\
\hline III & $17: 31$ & \multicolumn{2}{|l|}{3.94} & \multicolumn{2}{|c|}{-39.81} & 10.38 & \multicolumn{2}{|c|}{-16.08} \\
\hline Ia & $19: 41$ & \multicolumn{2}{|l|}{2.61} & \multicolumn{2}{|c|}{-67.65} & \multirow{2}{*}{$\begin{array}{l}6.82 \\
2.79\end{array}$} & \multicolumn{2}{|c|}{-48.77} \\
\hline $\mathrm{Ib}$ & $21: 12$ & \multicolumn{2}{|l|}{1.77} & \multicolumn{2}{|c|}{-86.34} & & \multicolumn{2}{|c|}{-71.24} \\
\hline
\end{tabular}

For comparison the satellite and sun positions were also included. A diagram of the results is given in Figure 6, where a polar projection is used to represent the field directions. To make possible the comparison between magnetic fields as observed in different regions we re-define the zero magnetic meridian to lie in the plane formed by the earth's magnetic polar axis and the radius vector of the satellite position. In this coordinate system the azimuthal variable of the field direction, $\phi$, is the angle whose magnitude is equal to the geomagnetic longitude of the field minus the geomagnetic longitude of the satellite position. 


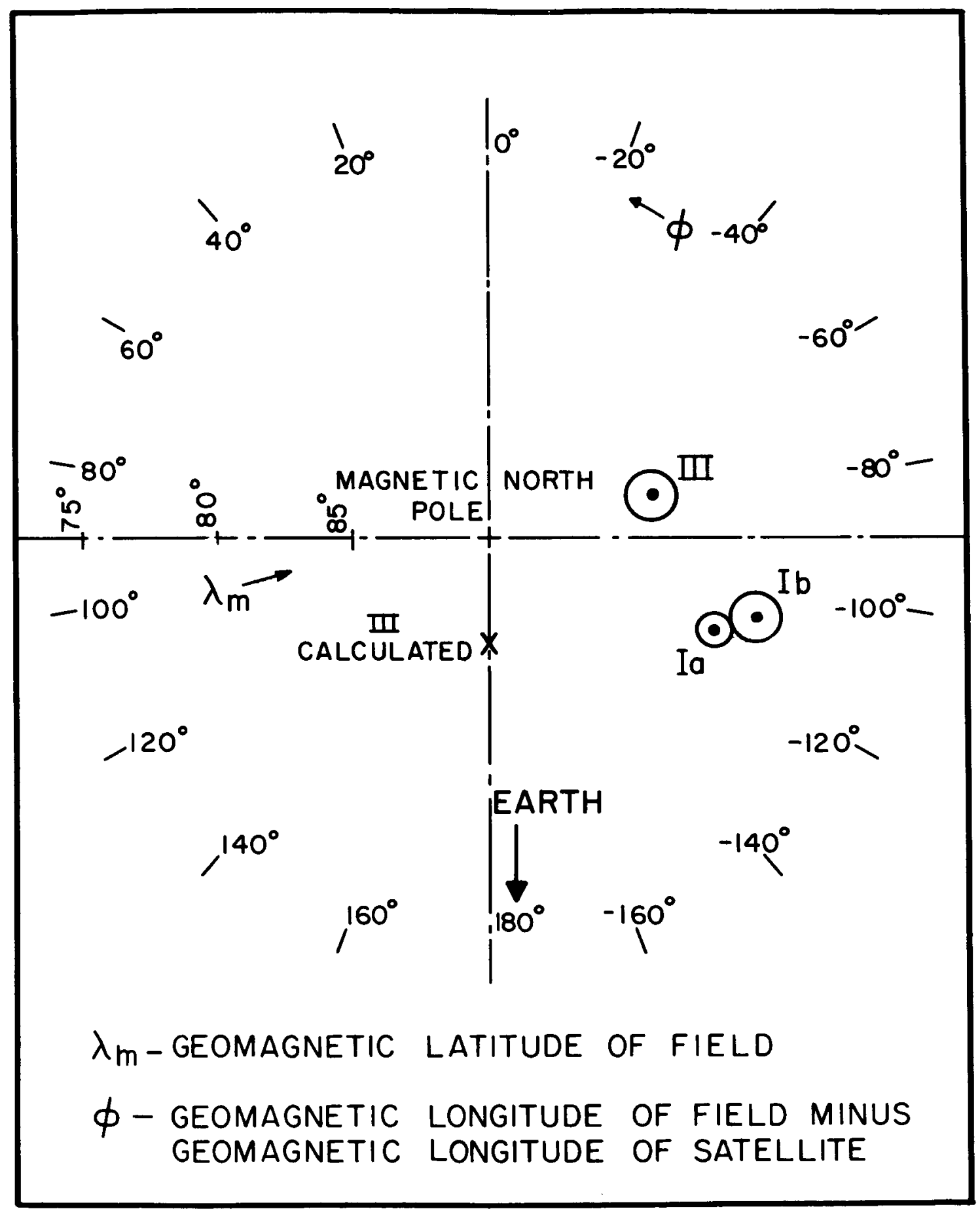

Figure 6-Polar plot showing the directions of the magnetic field in Regions la, $\mathrm{lb}$, and III. Also shown is the direction of the magnetic field as calculated under assumption described in the text. The azimuthal variable is the difference between the geomagnetic longitudes of the field and of the satellite. 
The points represent the directions of the magnetic field as observed in Regions III, Ia, Ib, and as calculated in Region III. The circles about the points correspond to standard deviations due to scatter of the raw data points. The lack of overlap of the data is evidence for slight temporal variations of the magnetic field. This was confirmed by taking several adjacent sets of data points in both Regions Ia and Ib. The results show a clustering of points with an average distance between the points of about two degrees.

The observed directions of the magnetic field seem to be out of phase with respect to the satellite by about $-90^{\circ}$ in the azimuthal direction rather than the expected $180^{\circ}$ if the field is calculated under assumptions stated before.

This difference does not seem significant. Even at 20,000 and 30,000 kilometers field measurements disagree by about 7 degrees of arc with a theoretical model based on the Jensen and Cain coefficients and the Mead cavity field. (Jensen and Cain, 1962; Mead, 1964). This disagreement may be due to slight uncertainties in the calibration of the instruments or an error in the assumed direction of the satellite spin axis, or both. The problem is currently being investigated.

The significance of these results is that in spite of several systematic uncertainties in the absolute measurement of the magnetic field and other effects such as the change in the satellite's geomagnetic latitude from $+3.94^{\circ}$ at $17: 31$ U.T. to $1.77^{\circ}$ at $21: 12 \mathrm{U} . \mathrm{T} .$, and the unknown spatial and temporal changes in the direction of the magnetic field, the observed field directions in Regions III, Ia and Ib can be fitted into a cone with a half angle of $3^{\circ}$.

This means that the magnetic field in Region III points almost in the same direction as the field in the trapping region and it is, therefore, reasonable to assume that Region III is just a temporary extension of the trapping region.

It is also possible to put a lower limit on the speed with which the trapping region overtook the satellite. The initial rise in the intensity of the magnetic field occurred at 17:30:52.9 U.T. At 17:31:04.3 U.T. the detector saw $140 \mathrm{kev}$ protons. During the $5.2 \mathrm{sec}$. counting period the detector could see protons with $90^{\circ}$ pitch angle. They were coming from directions shown in Figure 7. Since these protons were trapped their orbit could not extend beyond the boundary of the magnetic field. Thus we are led to the conclusion that during the interval of 11.4 seconds 


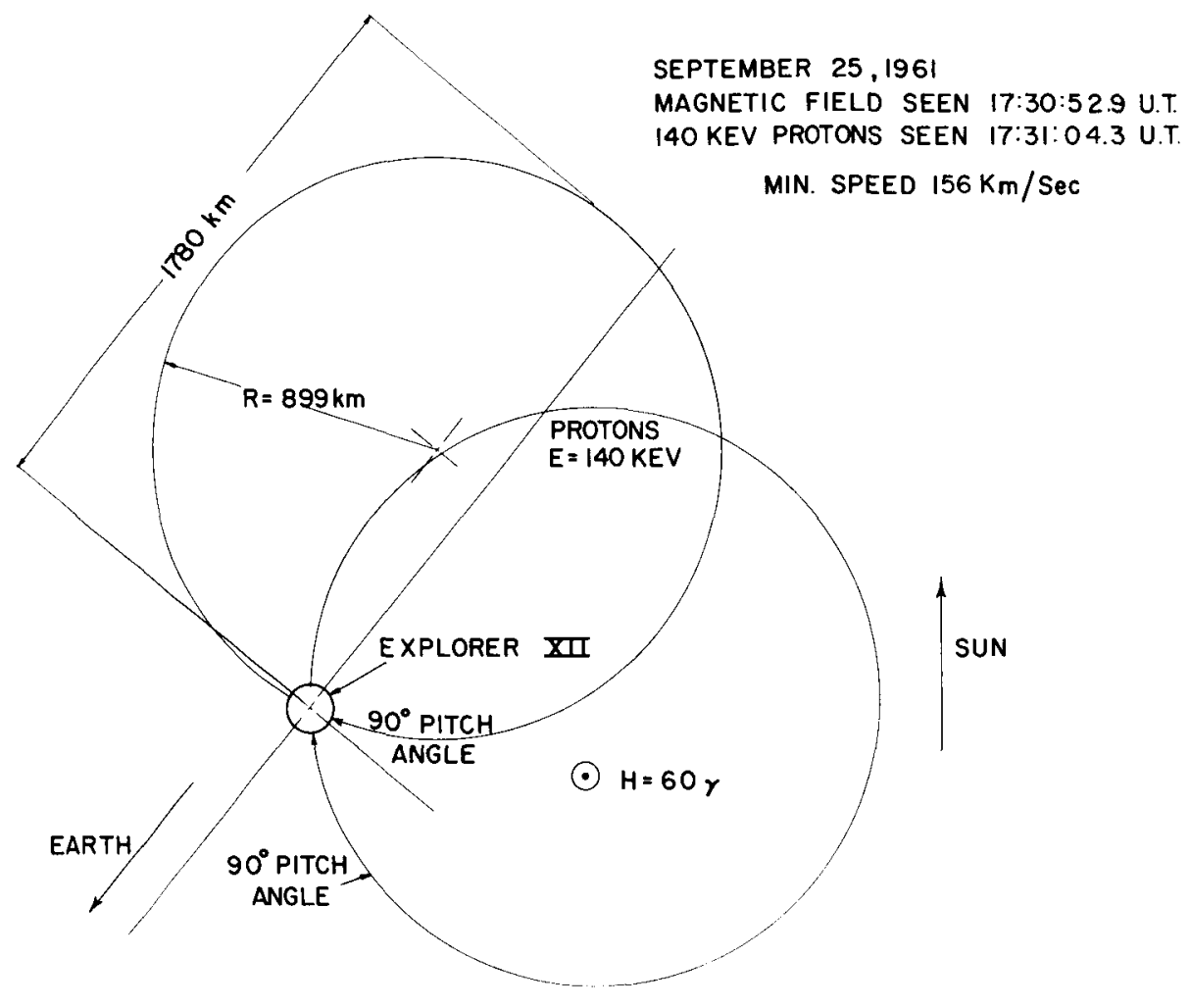

Figure 7-Orbits of trapped protons with $90^{\circ}$ pitch angle which were observed in Region III. At the time when the trapped protons were observed the boundary of the magnetosphere must hove been at least at a distance of $1780 \mathrm{~km}$ in the radial direction away from the satellite. The interval between the first observation of the increased magnetic field and the observation of $140 \mathrm{kev}$ protons was $11.4 \mathrm{sec}$. This puts a lower limit on the velocity of advance of the magnetosphere of 156 $\mathrm{km} / \mathrm{sec}$.

between the increase in the field and the appearance of $140 \mathrm{kev}$ trapped protons the boundary must have moved through a radial distance of at least $1780 \mathrm{~km}$. This puts a lower limit on its radial velocity of advance equal to $156 \mathrm{~km} / \mathrm{sec}$. A similar analysis can be performed in trying to estimate the speed of recession of the magnetosphere. The time interval between the instant when $200 \mathrm{kev}$ locally mirroring protons were seen and the instant when the field strength dropped to about 25 gamma was 29 seconds. To preserve closed orbits of trapped protons, the magnetosphere must have moved in that interval at least through 2130 $\mathrm{km}$. This puts a lower limit on the radial velocity of recession equal to $75 \mathrm{~km} / \mathrm{sec}$.

A survey of ground magnetograms was made. No identifiable features could be found in the records from low latitude stations. Large 
regular pulsation events were observed in two out of approximately twenty high latitude magnetograms which were surveyed. At Point Barrow (geomagnetic latitude $68.6^{\circ} \mathrm{N}$, geomagnetic longitude 241.2) the field vector began rotating counter-clockwise, as seen by an observer looking down at the earth, at about 17:31 U.T., or 0700 local time. The peak to peak amplitude was about 150 gammas and the period was 4 to 5 minutes. At Baker Lake (geomagnetic latitude $73.8^{\circ} \mathrm{N}$, geomagnetic longitude $315.2^{\circ}$ ) the peak amplitude was again about 150 gammas and the period was 4 to 5 minutes, but here the wave had a roughly linear polarization and began about 17:27 U.T., or 11:00 local time.

These ground records suggest that the observed rapid expansion and probable compression of the magnetosphere were associated with the excitation of a hydromagnetic disturbance in a thin shell of the outer magnetosphere. We do not, however, believe that the nearly simultaneous observation of one event on the satellite and of an event at two out of twenty ground stations can be interpreted as conclusive evidence that the two phenomena are related.

\section{INTERPRETATIONS}

We have argued that at 17:31 U.T. the magnetosphere expanded past Explorer XII at a velocity of at least $156 \mathrm{~km} / \mathrm{sec}$. Even if the external pressure dropped to zero, the plasma in the magnetosphere would not expand at a velocity greater than

$$
\mathrm{V}=\sqrt{\frac{\mathrm{B}^{2}}{4 \pi \mathrm{mn}}+\frac{2 \mathrm{c}^{2}}{\gamma-1}}
$$

where $\mathrm{n}$ is the number of protons per $\mathrm{cm}^{3}, \mathrm{c}$ is the speed of sound, and we will use $B$ equal to 60 gammas, $m$ equal to the mass of a proton, and $\gamma$ equal to $5 / 3$. The speed of sound in an ideal gas is given by

$$
\mathrm{c}^{2}=\frac{y \mathrm{RT}}{\mu}
$$

where $\mathrm{R}$ is the gas constant and $\mu$ is the molecular weight of the gas. Using these expressions, the expansion velocity is given by 


$$
\mathrm{V}^{2}=\frac{1.71 \times 10^{6}}{\mathrm{n}}+482 \mathrm{~T}
$$

where $\mathrm{T}$ is the temperature in electron volts and $\mathrm{V}$ is in $\mathrm{km} / \mathrm{sec}$.

If the magnetic energy density far exceeds the thermal energy density, the first term will dominate and an expansion velocity of 156 $\mathrm{km} / \mathrm{sec}$ implies a number density of less than 70 protons per $\mathrm{cm}^{3}$. If the magnetic field can be neglected, the second term dominates and implies a proton temperature of at least $50 \mathrm{ev}$. Several other possible combinations are listed in Table IV where it can be seen that either the number density must be less than about 100 protons per $\mathrm{cm}^{3}$ or the temperature must be greater than about 15 ev near the outer edge of the magnetosphere.

Table IV

\begin{tabular}{|l|c|r|r|r|r|r|}
\hline upper limit on $\mathrm{n}\left(\mathrm{cm}^{-3}\right)$ & $\infty$ & 341 & 174 & 117 & 88 & 70 \\
lower limit on $\mathrm{T}(\mathrm{ev})$ & 50 & 40 & 30 & 20 & 10 & 0 \\
\hline
\end{tabular}

After a time interval of 69 seconds a second boundary was observed. Several interpretations are possible. The observations could be caused by an expansion and subsequent compression of the entire magnetosphere, or a small portion of the magnetosphere; they could be caused by the expansion of a small portion of the magnetosphere which breaks off and continues to move outwards; they could be caused by a wave propagating in the surface of the magnetosphere. We cannot presently determine the cause of the observation.

\section{CONCLUSIONS}

On September 25, 1961 at 17:31 U.T. Explorer XII passed through a region of enhanced proton and electron intensity which lasted for 1 minute and extended over an apparent distance of $83 \mathrm{~km}$. At the same time the on board magnetometer registered an increase in the magnetic field.

Analysis of the data shows that the detected protons were trapped in the magnetic field. At the same time their cyclotron radii were 
much larger than the apparent width of the region. This fact points out the temporal character of the region. Similarities between the particle intensities and the strength and directions of the magnetic field in the region and in the outer belt suggest that the region comprises an impulse-like extension of the magnetosphere.

From knowing the time of the increase and the intensity of the magnetic field as well as the time when the $140 \mathrm{kev}$ trapped protons were detected, a lower limit on the outward radial velocity of the magnetospheric boundary can be determined to be $156 \mathrm{~km} / \mathrm{sec}$. Similarly the lower limit on the inward radial velocity of the magnetospheric boundary has been determined to be $75 \mathrm{~km} / \mathrm{sec}$.

The conclusions that can be drawn from our crude calculations a re that either the proton density near the outer edge of the magnetosphere is less than about 100 per $\mathrm{cm}^{3}$ or the temperature is greater than about $15 \mathrm{ev}$.

\section{ACKNOW LEDGMENTS}

We wish to express our appreciation to Dr. L. J. Cahill of the University of New Hampshire and to Mr. L. R. Davis and Mr. J. M. Williamson of the Goddard Space Flight Center for the use of their data from detectors flown on board Explorer XII. We also would like to thank Dr. Cahill and Mr. Davis for many helpful discussions.

\section{REFERENCES}

1. Beard, D. B., The Interaction of the Terrestrial Magnetic Field with the Solar Corpuscular Radiation, J. Geophys. Res., 65, $3559-3568,1960$.

2. Beard D. B., The Interaction of the Terrestrial Magnetic Field with the Solar Corpuscular Radiation, 2, second-order approximation, J. Geophys. Res., 67, 477-483, 1962.

3. Beard, D. B., and E. B. Jenkins, Correction to the Second Approximation Calculation of the Geomagnetic Field, Solar Wind Interface, J. Geophys. Res., 67, 4895-4896, 1962(1). 
4. Cahill, L. J., and P. G. Amazeen, The Boundary of the Geomagnetic Field, J. Geophys. Res., 68, 1835-1843, 1963.

5. Davis, L., Jr., and D. B. Beard, A Correction to the Approximate Condition for Locating the Boundary Between a Magnetic Field and a Plasma Field, J. Geophys. Res., 67, 4505-4507, 1962(1).

6. Davis, L. R., and J. M. Williamson, Low Energy Trapped Protons, Space Research, Proc. Intn'1 Space Sci. Symp., 3rd, Wa shington, D.C., 1962, North-Holland Publishing Co., Amsterdam, 1963.

7. Davis, L. R., and J. M. Williamson, Ion-Electron Detector, J. Geophys. Res., (to be published).

8. Hoffman, R. A., L. R. Davis, and J. M. Williamson, Protons of 0.1 to $5 \mathrm{Mev}$ and Electrons of $20 \mathrm{Kev}$ at 12 Earth Radii During Sudden Commencement on September 30, 1961, J. Geophys. Res., 67, 5001 5005,1962 .

9. Jensen, D. C., and J. C. Cain, An Interim Geomagnetic Field (abstract), J. Geophys. Res., 67, 3568-3569, 1962.

10. Mead, G. D., The Deformation of the Geomagnetic Field by the Solar Wind, J. Geophys. Res., 69, 1181-1195, 1964.

11. Mead, G. D., and D. B. Beard, The Shape of the Geomagnetic Field Solar-Wind Boundary, J. Geophys. Res., 69, 1169-1179, 1964.

12. Spreiter, J. R., and A. Y. Alksne, On the Effect of a Ring Current on the Terminal Shape of the Geomagnetic Field, J. Geophys. Res., $67,2193-2205,1962$.

13. Spreiter, J. R., and B. R. Briggs, Theoretical Determination of the Form of the Boundary of the Solar Corpuscular Stream Produced by Interaction With the Magnetic Dipole Field of the Earth, J. Geophys. Res., 67, 37-51, 1962 .

14. Spreiter, J. R., and B. J. Hyett, The Effect of a Uniform External Pressure on the Boundary of the Geomagnetic Field in a Steady Wind, J. Geophys. Res., 68, 1631-1642, 1963. 